BULLETIN Bulletin hispanique

HISPANIQUE Université Michel de Montaigne Bordeaux

115-1 | 2013

Poésie et société en Espagne : 1650-1750

\title{
Los inicios de la investigación sobre el arabismo léxico en español
}

El Compendio de Francisco López Tamarid frente a la Recopilación de Diego de Guadix

\section{Stefan Ruhstaller}

\section{(2) OpenEdition}

Journals

Edición electrónica

URL: http://journals.openedition.org/bulletinhispanique/2475

DOI: 10.4000/bulletinhispanique. 2475

ISSN: 1775-3821

Editor

Presses universitaires de Bordeaux

Edición impresa

Fecha de publicación: 1 junio 2013

Paginación: 253-270

ISBN: 978-2-86781-898-1

ISSN: 0007-4640

Referencia electrónica

Stefan Ruhstaller, «Los inicios de la investigación sobre el arabismo léxico en español », Bulletin hispanique [En línea], 115-1 | 2013, Publicado el 01 junio 2016, consultado el 01 mayo 2019. URL http://journals.openedition.org/bulletinhispanique/2475; DOI : 10.4000/bulletinhispanique.2475 


\title{
Los inicios de la investigación sobre el arabismo léxico en español. El Compendio de Francisco López Tamarid frente a la Recopilación de Diego de Guadix
}

\author{
Stefan Ruhstaller \\ Universidad Pablo de Olavide - Sevilla
}

Les deux auvres lexicographiques les plus anciennes consacrées aux arabismes en espagnol, élaborées en moins de dix ans à la fin du XVI siècle, ont surgi du même cercle social et géographique. L'analyse comparative détaillée montre des affinités nombreuses et voyantes, mais aussi des différences remarquables, et permet de réfléchir au contexte culturel qui a favorisé l'apparition d'un genre particulier dans l'histoire de la lexicographie espagnole.

Mots-clés : lexicographie espagnole, analyse comparative, arabismes en espagnol, étymologie, identité culturelle.

Las dos obras lexicográficas más antiguas sobre arabismos en español fueron elaboradas en un periodo de menos de diez años al final del siglo XVI y surgieron del mismo círculo social y geográfico. El análisis comparativo revela numerosas afinidades, pero también diferencias significativas, y nos permite reflexionar sobre el contexto cultural que estimuló la aparición de un tipo peculiar de obra en la historia de la lexicografía del español.

Palabras claves: Lexicografía española, análisis comparativo, arabismos en español, etimología, identidad cultural.

The two oldest works on lexical elements of Arabic origin in Spanish, written within the interval of less than a decade at the end of the 16th century, arose from the same social and geographical circle. Our detailed comparative analysis reveals numerous affinities, but also remarkable differences, and allows us to reflect on the cultural context that stimulated the appearance of a particular type of dictionary in the history of Spanish lexicography.

Keywords: Spanish Lexicography, comparative analysis, Arabism in Spanish, etymology, cultural identity. 


\section{Dos INTÉRPRETES COETÁNEOS DE LA INQUISICIÓN GRANADINA,} AUTORES DE LAS PRIMERAS OBRAS LEXICOGRÁFICAS SOBRE ARABISMOS

A finales del siglo XVI fueron elaboradas, con muy pocos años de diferencia, dos obras lexicográficas de innegable interés para la historia de la lengua española y la lexicografía histórica del español: por una parte, el Compendio de algvnos vocablos arábigos introdvzidos en la lengua caftellana en alguna manera corruptos, de que comúnmente vfamos, pueftos por orden alphabético (1585), de Francisco López Tamarid, y por otra parte, la Recopilación de algunos nombres arábigos que los árabes (en España, Francia, y Italia) pusieron a algunas ciudades y a otras muchas cosas (1593), de Diego de Guadix. Las dos versan sobre materia prácticamente idéntica: la recopilación y explicación etimológica de nombres tanto comunes como propios de presunto origen árabe, en uso principalmente en castellano. Pero las afinidades entre ambas obras van más allá: las dos reflejan una perspectiva claramente centrada en Granada (especialmente en lo referente a la selección de los nombres de lugar interpretados), y las dos son obra de religiosos que vivieron en dicha ciudad y ejercieron la labor de intérprete de lengua árabe en el Tribunal de la Inquisición ${ }^{1}$.

Aun siendo evidentes las afinidades, se perciben de inmediato también notables diferencias, principalmente en lo relativo a la amplitud de las dos obras: la de Guadix incluye en su macroestructura 4336 voces, distribuidas entre 2275 apelativos y 2061 nombres propios (Moreno 2007: XLVI), mientras que la de Tamarid comprende un total de 603 lemas, de los cuales 532 figuran en una primera parte centrada de forma casi exclusiva en apelativos, y 76 en una segunda, titulada

Exposición la más fiel y cierta que fe ha podido hazer de los nombres aráuigos y del verdadero fignificado que tienen en nueftro romançe caftellano: aßí los que fe vfan en la ciudad de Granada como en otras ciudades y villas de Efpaña en puertas, plaças, calles, ríos, montes, como en otros lugares particulares.

y dedicada íntegramente a nombres de lugar. Por otra parte, la información que ofrece Guadix en su microestructura es muy variada y detallada, mientras que Tamarid se limita por lo general a explicar el significado romance de los arabismos de forma escueta y con escasa precisión. Guadix logra el mayor volumen de la macroestructura gracias a una actitud mucho más "generosa» a la hora de considerar posible un origen árabe en el caso del léxico general, y en el caso de los nombres de lugar gracias a una labor de recopilación sistemática, restringida en mucha menor medida a Granada. Y las diferencias trascienden el propio contenido de las dos obras, ya que estas divergen radicalmente también

1. En el propio texto del Compendio, este se atribuye al «illuftre feñor Francifco López Tamarid, racionero de la fancta yglefia de Granada, familiar y intérprete de la lengua arábiga en el fancto Officio». De Diego de Guadix consta que fue nombrado para el cargo el 22 de septiembre de 1587 (Bajo / Maíllo 2005: 16). 
en cuanto a su difusión posterior: mientras el Compendio de Tamarid apareció impreso como apéndice a la edición granadina de 1585 del Diccionario de romance en latín de Nebrija, y reeditado posteriormente en diversas ocasiones (hecho que facilitó una cierta repercusión en los diccionarios y los estudios lexicológicos posteriores), la Recopilación de Guadix, redactada durante la estancia de este en Roma, ha permanecido en estado manuscrito hasta nuestro siglo, cuando Elena Bajo Pérez y Felipe Maíllo Salgado por una parte, y por Águeda Moreno por otra, publicaron dos ediciones independientes ${ }^{2}$, rescatando así la obra de un olvido casi absoluto (el único autor que la había utilizado con cierta regularidad fue Covarrubias).

La pregunta que se impone es: ¿existe una relación entre las dos obras? En caso afirmativo: ¿en qué consiste, y cómo se manifiesta? Una posible respuesta ha de buscarse a través de la comparación de los propios textos, ya que no contamos con datos externos claros. De hecho, Diego de Guadix no hace referencia alguna a su precursor y paisano, a pesar de que, al final de su obra, se declara explícitamente deudor de otros arabistas (Bajo / Maíllo 2005: 1037):

Estos nombres arábigos y la interpretación dellos an sido comunicados con muchos hombres y muy doctos en lengua arábiga, y particularmente en esta sancta ciudad de Roma, e comunicado algunos dellos con el reverendo señor don Leonardo Abel, maltés y obispo de Sidón, y con fray Thomás de Terrachina, frayle de la sagrada religión de sancto Domingo, y con Juan Baptista Raymundo, y con Pablo Ursino, y con Guillermo Ursino, que, como hombres que en esta sancta ciudad de Roma son del cabildo o congregación de la lengua arábiga, y tan peritos en la lección de libros de la dicha lengua arábiga, pudieron dar su parecer y aun advertirme de algunos nombres de que yo no me acordaua y con cuya declaración yo no acabaua bien de atinar.

Y no solo esto: como ya destacó Moreno (2006: 1183), Diego de Guadix, en el prohemio a su obra, justifica ésta alegando precisamente la inexistencia de tratados sobre el tema (afirma explícitamente que «no ay libros que poder leer y consultar que traten d'este menester»). Por su parte, Tamarid, quien pertenecía indudablemente al mismo círculo erudito granadino, omite cualquier referencia a Guadix a pesar de que menciona a otras personas que colaboraron con él en la confección del Compendio, según veremos más adelante.

Ya los editores de la Recopilación se plantearon la posibilidad de una relación entre las dos obras. Sus breves pesquisas al respecto, no obstante, los llevaron a conclusiones contrapuestas. Por una parte, Moreno (2006: 1183-1184) ilustra su juicio de que «la distancia entre ambas obras es ingente» reproduciendo tres entradas concretas (los apelativos abismales, faysán y panilla) que, en efecto, no presentan similitud alguna, y concluye que, «si bien es cierto que la mayoría de voces del listado de Tamarid están incluidas en el corpus macroestructural de la Recopilación», Guadix «no le debe nada a Tamarid, ni en cuestión de material y, mucho menos, en cuestión técnica.» Muy diferente es la opinión de Bajo / Maíllo (2005: 21-22), quienes, partiendo del hecho de la proximidad

2. Citaremos la obra a través de la edición de Bajo / Maíllo 2005, impecable en todos los sentidos. 
personal de ambos autores (Tamarid «era también familiar de la Inquisición en Granada»), afirman, basándose en un cotejo más amplio que el llevado a cabo por Moreno, que «Guadix seguramente conocía de sobra la obra de López Tamarid», si bien diferencian entre la parte dedicada a los apelativos -que «solo pudo ser de utilidad de cara a la macroestructura y solo limitadamente» (2005: 27) - y la dedicada a toponimia; en cuanto a esta última consideran «más que probable $[\ldots]$ que Guadix haya tenido en cuenta al menos la "Exposición" que figura al final del Compendio (1585) de López Tamarid: puede haber tomado de esa sección algunos hidrónimos y algunos microtopónimos, como, por ejemplo, los nombres de puertas, plazas y calles de Granada» (2005: 22); recalcan en otro lugar de su estudio que "con certeza esta parte le fue utilísima a Guadix, aunque López Tamarid solo ofrezca informaciones muy escuetas y aunque el número total de voces sea insignificante en comparación con el que encontramos en la Recopilación» (2005: 27).

En vista de estas opiniones discrepantes, y dado el valor que indiscutiblemente tienen las dos obras, tanto intrínsecamente como por su relevancia en la historia de las ideas lingüísticas y de la lexicografía del español, bien merece la pena indagar sobre una posible relación entre ellas y sobre el contexto cultural en que surgieron.

\section{ANÁLISIS COMPARATIVO DE LAS DOS OBRAS LEXICOGRÁFICAS}

\section{II.1 El léxico incluido en las macroestructuras}

La obra de Tamarid se divide, como ya señalamos, en dos partes independientes de extensión muy desigual; el criterio en que se basa esta división es, obviamente, el de la distinción entre nombre común y nombre propio, aun cuando su aplicación no fue del todo sistemática, ya que en la primera, como ya advirtieron Bajo / Maíllo (2005: 27, n. 7), se deslizó una corta serie de nombres propios ${ }^{3}$. Cada una de estas partes, a su vez, se halla organizada según principios propios; en la dedicada a los apelativos, estos aparecen ordenados alfabéticamente, si bien en tres nomenclaturas separadas: una primera, que abarca cerca del $97 \%$ del material, comprende voces cuyo uso no se limita

3. A los casos identificados por estos autores -Albaida, Alcántara, Alcantarilla, Alcoba, Alcaná, Algeziras, Algar, Almedina- podemos añadir, al menos, los de Alhambra, Çocodover y Tánjar. El único antropónimo incluido erróneamente en la primera parte del Compendio, Maluco Mulei, figura también (si bien bajo la forma simple Maluco) en Guadix; este justifica explícitamente su decisión de incluir la voz: "Declararlo é por ser tan nombrado entre christianos, después de aquella batalla de África donde murió don Sebastián, rey de Portogal». Para la cuestión que nos interesa en este estudio, la de una posible influencia de la obra de Tamarid en la Recopilación, puede ser significativo el hecho de que ninguno de los topónimos que se habían infiltrado en la primera parte del Compendio sea considerado apelativo por Guadix. Un caso especial es el registro Ceca, que Tamarid únicamente explica como topónimo cordobés: «Es casa de deuoción de Cordoua, donde venían los Moros.» Guadix, en cambio, define la voz con el valor apelativo que aún hoy tiene en castellano. 
geográficamente de forma expresa; una segunda se centra en «nombres arávigos vsados en el Reyno de Murcia» (ocho voces); y una tercera está constituida por nueve «nombres arávigos en Portugal». En la parte onomástica (Exposición), en cambio, el criterio de ordenación empleado es de índole puramente geográfica: un primer conjunto de 29 lemas está formado por nombres referentes a sitios de la ciudad de Granada o su inmediato entorno, subclasificados como nombres de puertas ${ }^{4}$, plazas, calles y ríos. Los restantes 47 corresponden a toponimia de otras regiones de España, aunque también aquí se constata la perspectiva geográficamente muy limitada de la obra, ya que más de la mitad del material corresponde al Reino de Granada; de nuevo se subcategorizan los nombres según el tipo de referente.

Frente a esto, Guadix incluye en una única macroestructura, dispuesta en su integridad por orden alfabético, indistintamente nombres comunes y nombres propios; entre estos últimos figuran topónimos de todo el mundo entonces conocido (incluso algunos del norte de Europa y de América), si bien los materiales más ricos y originales corresponden, al igual que en el caso de la Exposición, al Reino de Granada.

\section{II.1.1 El léxico común}

El más de medio millar de voces romances de presunto origen árabe recopilado en la primera parte del Compendio constituye un corpus nada despreciable que podía tener una gran utilidad para Guadix, sobre todo si tenemos en cuenta que, a pesar de haber anunciado en el preámbulo su propósito de compendiar «algvnos vocablos de que comúnmente vsamos», Tamarid había ido mucho más allá, puesto que había recogido también numerosas voces que no gozaban de difusión general, y que ni siquiera él, un granadino culto de la segunda mitad del siglo XVI, familiarizado además con la cultura morisca, empleaba como hablante ni conocía de primera mano. Las marcas lexicográficas con que Guadix -hablante con una competencia lingüística en castellano idéntica a la de Tamarid- restringió el uso de buena parte de este léxico son elocuentes al respecto: limita explícitamente en lo diatópico cerca de cien voces que comparte con Tamarid. Otros arabismos recogidos en el Compendio estaban sometidos a una restricción diatécnica: pensemos en voces del lenguaje militar (como abismales), del comercio de la seda (almotalefe, azache, geliz), la cetrería (bahari, borni), la navegación (lebeche), la albañilería (iorfe), etc. Buena parte de este léxico gozaba de un uso muy restringido en lo diastrático al tratarse de voces que designaban referentes propios y específicos de la cultura tradicional de la comunidad morisca que no habían trascendido apenas del habla de este sector de la sociedad del s. XVI:

zarzahán. Es cierta seda de labores que texen los moros.

maginacete o machumacept. Letuario que hazían los moriscos para ahíto hecho de siete cosas.

moxí. Es cierto género de caçuela quajada, de que vsauan los moros.

4. Del escaso rigor de esta ordenación da una idea el hecho de que en el apartado «Pvertas de Granada» se incluyan registros como "Puerta Bisagra en Toledo» o "Puerta Balnadú en Madrid». 
Como se desprende del frecuente uso del imperfecto en las definiciones, bastantes de los arabismos reunidos habían ya caído en desuso a finales del s. XVI, hecho señalado a veces incluso de forma explícita por Guadix 5 .

De todo lo anterior concluimos que el material reunido por Tamarid, a pesar de la apariencia poco elaborada de la obra, era fruto de una búsqueda plenamente consciente que había implicado la consulta de fuentes externas. Con todo, el material acopiado por Guadix es aún mucho más amplio: según el recuento de Moreno (2007: XLVI), la Recopilación incluye 2275 artículos dedicados a voces del léxico general, cuadruplicando así las entradas del Compendio. El hecho de que Guadix lograra constituir una macroestructura tan superior se explica por varias razones. En primer lugar, no se limitó al léxico del castellano y del portugués, como había hecho Tamarid, sino que recogió también bastantes palabras sobre todo del italiano, pero también del francés, del turco, del maltés, e incluso del latín ${ }^{6}$. En segundo lugar, tuvo un criterio mucho más "generoso» a la hora de identificar las voces por su origen presuntamente árabe: su "propensión innegable», en palabras de Bajo / Maíllo (2005: 27, n. 7), «a encontrar arabismos por todas partes», le llevó a incluir numerosísimas voces de etimología claramente no árabe; nos ilustran esta tendencia artículos como el dedicado a fraile, cuyo origen romance es negado explícitamente y contra toda evidencia por Guadix:

Vien ha pensado el mundo qu'este nombre frayle y freyle son corrupciones d'este nombre latino fratre, y no es sino una corrupción d'este nombre fayleçeuf, que en arábigo significa filósofo. [...] este nombre frayle o freyle, para hazerle significar en femenino, con mudar la .e. en .a. está todo negociado; esto digo porque en Portogal llaman a las monjas por este nombre freylas...

No obstante, otros muchos registros prueban que Guadix había llevado a cabo una búsqueda totalmente independiente del Compendio que le proporcionó no pocos arabismos verdaderos, algunos de uso muy limitado, como alhurreca, aljabibe, alhaqueque, algorfa, alfayda, almocadén, etc. Además, en los registros comunes a ambas obras, la información original que expone Guadix revela que había logrado reunir información mucho más rica que Tamarid: por ejemplo, el murcianismo abacora, explicado escuetamente como "Son brebas» en el Compendio, se describe en la Recopilación (donde se lematiza como albacora y bacora) con interesantes detalles, sobre todo de tipo dialectológico:

\footnotetext{
albacora. Llaman en algunas partes d'España, .i., en el reyno de Murcia, a una suerte de higos gruesos y tempranos que son los primeros que dan las higueras. [...] En otras partes d'Espańa llaman a esta suerte de higo breba; y en una parte de Estremadura, a que llaman Jerez de los Caualleros, la llaman vacalar.
}

5. Comenta acerca de aljófar: «En Italia llaman a estos granillos perlas, y aun en muchas partes d'España, dando de mano al nombre arábigo aljófar, los llaman también perlas.» Alguna de las voces registradas incluso designaba un referente ajeno a la realidad cultural de la Península, como aduar («Es abitación de alárabes»).

6. La distribución por lenguas puede consultarse cómodamente en la ed. de Bajo / Maíllo 2005: 1041-1084. 
bacora. Llaman en algunas partes d'España a una suerte de higos tempranos. Véase el nombre albacora.

Para determinar si Guadix utilizó el Compendio como base para extraer material léxico puede aportarnos indicios una comparación de los dos conjuntos de lemas. Ya Bajo / Maíllo (2005: 27, n. 7) cotejaron ambas macroestructuras al menos parcialmente y seńalaron toda una serie de voces recogidas por Tamarid que faltan en la Recopilación: abucasta, açofeifa, albihares ${ }^{7}$, agujeta, albarca, alcorán, alfaquí, algaida, aljama, almedano, aloxa y alquermes. ${ }^{8}$. Para completar la lista podemos añadir las siguientes voces: aluarazos, algarvè, almuédano, arriate, atayfor, axarafe, azache, balat, badana, bruneta, carmesi, çanahoria, çaga, cañuto, castaña, çabila, clauellina, cosquillas, confites, cozina, espelta, faxa, gaita, gálgana, garrapata, gorguera, gorjal, gorra, guida, hacino, hilachas, iorfe, iuglar, ladilla, lampazo, albudeca, mingrana, acril o aqueril, romana, mancaz, milla, morado, melón, nutria, oro de Tíbar, porra, pujabante, pulga, regayfa, resina, ribadoquin, tertil, tarbea, tagarote, topo, torçuelo, xarifa. Otros lemas de Tamarid no figuran como tales en la macroestructura de Guadix, aunque sí se señalan en el interior de otros artículos: algarroba (recogido s.v. algarrovo), algebrista (s.v. álgebra), azeytuna (s.v. azeyte), bacía (s.v. bacín), baho (s.v. bahear), barrenar (s.v. barrena), capuz (s.v. capa), chanqueta (s.v. chanca), garramar (s.v. garrama), guillote (s.v. guilla), marchamo (s.v. marchamar), ronda (s.v. rondar), saya (s.v. sayo). Es cierto que no podemos negar la posibilidad de que en algún caso Guadix omitiera determinados lemas de Tamarid por considerarlos de etimología no árabe ${ }^{10}$; no obstante, el franciscano, tan empeñado en recopilar un número máximo de arabismos, difícilmente habría pasado por alto tantas voces identificadas ya por Tamarid como de origen árabe si las hubiera tenido a mano, pues, a juzgar por su forma de establecer etimologías, fácilmente habría encontrado hipótesis árabes para la inmensa mayoría de ellas. Es importante destacar, además, que estas ausencias no solo son relevantes desde el punto de vista cuantitativo, sino también desde el cualitativo, ya que los datos de Tamarid que no reencontramos en la Recopilación a menudo son de naturaleza muy peculiar: tecnicismos de difusión diatópica muy restringida (como tertil "derecho de la seda"), dialectalismos propios de zonas alejadas de

7. Esta es la forma que realmente figura en Tamarid, no albibares como recogen Bajo / Maíllo: 27 n. 7.

8. El registro alexía "puches", reseñado igualmente como ausente en la Recopilación por Bajo / Maíllo, en cambio, sí figura ahí, si bien bajo la forma alexixa.

9. Incluyo esta voz porque en Guadix figura no algarve "poniente", sino su homónimo algarve "cueva".

10. De hecho, excluye la voz gaita porque no la derivaba del árabe, como manifiesta explícitamente en su comentario dedicado a albogue: «An deseado, en España, dar de mano a esta algarabía y llamar y nombrar a esta dicha flauta por nombre castellano, y la an baptizado por este nombre: gayta çamorana.» 
Granada (almedina ${ }^{11}$, algaida ${ }^{12}$, acril, o aqueril, axarafe), y, en general, voces escasísimamente atestiguadas por los lexicógrafos (calahorra "fortaleza"; hafiz "guarda de la seda"13; iorfe "pared hecha de piedra seca"14; mancaz).

Por otra parte, el cotejo de las dos macroestructuras revela la existencia de numerosas diferencias formales entre los lemas comunes a ambas obras. No me refiero a las divergencias de carácter meramente gráfico (Bajo / Maíllo 2005: 27, n. 7 , señalan una serie de ejemplos), de escasa relevancia en una época de tanta inestabilidad en este terreno como la que nos ocupa, sino a aquellas que son reflejo de variantes fonéticas reales; a los casos recogidos por Bajo / Maíllo (2005: 27, n. 7) -azial / aziar, adalid / adalí y adalil, alcoscuçú / alcozcuçu, alfiler / alfilel, aljonjolí / aljonjolin, almarraza / almarraxa, almádena I almádana, almotalafe / almotalefe, assarrabacar / asarabassar- podemos añadir los siguientes (indico en primer lugar la forma de Tamarid): azaguán / açaguán o zaguán, açutea laçotea o açutea, adalid / adalil, alcaduz / arcaduz, alcahuete I alcagüete, alfiler / alfilel o alhilel, álgebra / álgebra o álquebra, alcácer / alcáçar, aguinaldo / aguilando, alcuña / alcuña o acuña, aljonjolí lajonjolí, alexía I alexixa, alizar / alizer o alizar, almoradux / almoradux o amiradux, almohada I almofada, almirez I almihirez, almandarahe / almandarache, anacales / añacal o hañacal, alquería I alcaría, alquilé I alquilé o alquiler, arfil Iarfil o alfil, ataúd lataut $o$ ataúd, aulaga laulaga o aliaga o abolaga, axarave I xarave, aximenez («Es solana») / axumez («Llaman en algunas partes d’España a un balcón o corredorcillo que buela sobre unos canes, fuera del hilo de la pared, para en él tomar y gozar del sol»), çaragüel / çaragüeles o çaragüelles, ceifa / çayfa, ceifar / çayfar, fanal / fanal o fanar, gebeli puerco / gebeli o puerco jabali, hanega / hanega o fanega, magazen / almacén, matalahúga I matalahúva, magrán / magram, nebli I nebli o ñebli, oraçuz loroçuç, quintal / quintar o quintal, tabaque I tabaque o atabaque o altabaque, telliz / teliz. Otras diferencias no se deben a la elección de una variante fonética, sino a la de una forma diferenciada morfológicamente: acelgas I acelga, alcahueta / alcagüete, alforjas / alforja, albur / albures, alharaca I alharacas, acicates lacicate, axorca / axorcas, caracoles / caracol, espárragos I espárrago, matracas / matraca, mocos / moco, naipes / naype; también alfahar "ollería” / alfahar o alfaharero ("Llaman en España al oficial a que por nombre castellano llaman ollero o cantarero»), banco / banca o banco, naranjo / naranja y naranjo. En ocasiones, Guadix lematiza el arabismo conjuntamente con las demás palabras con que constituye una ampliación sintagmática: carcajadas I carcajadas de risa, mazari I mazari o ladrillo mazarí, moxi I moxi o caçuela moxí, sirga / sirga o llevar a la sirga, tigual / tigual o la renta del tigual.

11. Arabismo propio del portugués, y documentado, así mismo, en un documento murciano alfonsí (Corriente 1999: 189).

12. Dialectalismo limitado a las hablas andaluzas más occidentales y atestiguado principalmente en toponimia ( $D C E C H$, s.v.).

13. Calificada en el $D C E C H$ de "palabra rara".

14. El $D C E C H$, que desconoce el registro de Tamarid (da como primera documentación el diccionario de Oudin), comenta: «Palabra muy rara en castellano.» 
Es cierto que estas diferencias entre lemas no descartan necesariamente la posibilidad de que Guadix se basara en el Compendio, ya que pueden ocasionalmente ser fruto de una decisión meditada: el franciscano pudo dar preferencia a una forma más recomendable desde el punto de vista de su uso, o más cercana al presunto étimo ${ }^{15}$; también sería fácil de entender, por ejemplo, que no reprodujera una forma que constituye una errata como galeza en lugar de gazela. No obstante, sumadas a la ausencia en la Recopilación de numerosos lemas llamativos registrados por Tamarid, estas discrepancias formales nos obligan a extraer la conclusión de que Guadix con toda probabilidad no aprovechó el Compendio de forma directa durante la redacción de su obra.

\section{II.1.2 El material toponimico}

La inmensa mayoría de los topónimos registrados en la Exposición está presente también en la nomenclatura de la Recopilación: de los 75 nombres recogidos en la primera, 69 -esto es, un 92\%- son objeto de interpretación etimológica igualmente en la segunda. A priori, la amplia coincidencia podría explicarse como resultado de un trasvase directo de datos de una obra a otra, y la no inclusión de seis nombres en parte como resultado de una criba consciente ${ }^{16}$, en parte como omisión involuntaria y casual en el traslado de material desde la fuente ${ }^{17}$.

Sin embargo, dichos 69 nombres no constituyen sino una mínima parte (un $3,34 \%$ ) del conjunto de los nombres propios recopilados por Guadix, quien había llevado a cabo una búsqueda mucho más sistemática, entre otras regiones precisamente en la de Granada, que conocía personalmente tan de cerca como Tamarid $^{18}$. Por otra parte, descubrimos, de nuevo, numerosas diferencias formales entre los nombres lematizados en una y otra obra. Al igual que en el caso de los apelativos, hemos de distinguir entre divergencias meramente gráficas (Bibalbonut / Bibalbonud, Río de Genil/ Xenil, etc.), de escasa relevancia, y discrepancias que derivan de pronunciaciones diferenciadas: algunas son de poca entidad, como Ginalariph / Genalarife o Ginalarife, Guadalet / Guadalete, otras, sin embargo, reflejan formas más distantes que, o bien revelan la coexistencia de variantes fonéticas reales en el habla de la época (Rahbadbedis I

15. Así, lematiza la extraña variante aguilando por ser más cercana al étimo árabe que postula para la voz aguinaldo (forma que rechaza expresamente), o albures por considerar que el étimo árabe es un plural. Sin embargo, Guadix no es, ni mucho menos, sistemático en este sentido, pues a menudo lematiza formas que después condena explícitamente en el interior del artículo (pueden verse ejemplos representativos s.vv. arcaduz, gebeli o puerco jabali, y quintar o quintal).

16. Por ejemplo, pudo excluir formas como Escurial o Álava por considerar que no tenían origen árabe.

17. Así se justificaría la exclusión de nombres tan ligados a su tierra natal como Mavror, Puerta de Alcaçava, Baça, para los que sin duda fácilmente habría encontrado un étimo árabe. El sexto nombre no incluido es Gabalchol, que no he podido localizar.

18. La enorme superioridad en materia documental de Guadix queda demostrada no solo por la cantidad de los nombres recopilados, sino también por la abundante información adicional que ofrece acerca de estos, según veremos en 2.2.2. 
Rahbalbedis, Belmar / Belmar o Bedmar, Daraçultan / Daracután, Guadalerze / Guadalhorçe o Guadalherça), o bien son fruto de una transcripción defectuosa por parte de uno de los dos lexicógrafos: en los casos de Çacatim / Çacatín y Guadarricaz / Guadarriçaz ${ }^{19}$ es sin duda Tamarid el que consigna una forma errónea, mientras que en los de Xarragui / Xaragüi o El Xaragüi o Fajalausa / Frajaleuz ${ }^{20}$ habría que disponer de documentación histórica adicional para determinar quién se ajusta más a la realidad lingüística de la época. En algunos casos no cabe duda de que Guadix transcribe una forma discrepante del uso real bajo la influencia del étimo árabe que establece: Bibarreha / Bibalreha, Campo de Çafayona / Çafalayona (< fahç al 'oyona "campo de nuestras fuentes").

En suma: el número de discrepancias es sin duda lo suficientemente alto, y sus características lo suficientemente reveladoras, como para convencernos, nuevamente, de que Guadix no tuvo delante el texto de Tamarid mientras redactó su Recopilación.

\section{II.2 Comparación de las microestructuras}

\section{II.2.1 Léxico común}

La microestructura de la parte dedicada al léxico común del Compendio se caracteriza por su falta de sistematicidad y su desarrollo rudimentario. La mayoría de las voces recogidas (277 de 532) no van acompañadas de información alguna, lo cual convierte la obra hasta cierto punto en un mero listado de voces seleccionadas según el criterio de su presunto origen árabe. En las restantes 255 el elemento más constante es la información semántica. Pocas veces cabe hablar de verdaderas definiciones, pues prevalecen las explicaciones escuetas y aproximadas mediante palabras semánticamente cercanas sin llegar a ser sinónimos (alcandora "Es camisa», añafil «Es trompeta») o mediante hiperónimos (abucasta "Es aue», alcotán "Es género de halcón», axaraue "Es beuida»). En ocasiones los comentarios tan solo sitúan la voz en un contexto de uso (cartabón "Es de medidores de tierra»; roque "Es de axedrez»; alfarje «Es de molino de azeyte»), o aluden vagamente al referente (buxahamel

19. Se trata del nombre de un pequeño río al norte de la Sierra Morena jiennense conservado hasta hoy como Guadarrizás o Guarrizás, formas que demuestran que la grafía Guadarricaz de Tamarid es errónea; el hecho de que Guadix no reproduzca este error indica que no dependió del Compendio como fuente. El caso contrario es Calatançón, forma disparatada que Guadix habría transcrito correctamente como Calatañaçor de haber contado con el Compendio (donde figura correctamente). Otros lemas recogidos bajo formas discrepantes difieren desde el punto de vista morfológico: Hatabin o El Hatabin se opone a un simple Hatabin en Tamarid. Por otra parte, este autor recoge una forma sin duda antigua en Loxa, "Llaman Hiznaloxa, que es el castillo de las losas", que Guadix difícilmente habría desaprovechado de haber utilizado el texto de forma directa. En el caso de la pareja Puerta de Eluira / Bibalbira el criterio de lematización discrepa aún más, pues parece que Guadix optó, en contra de lo habitual, por lematizar una forma árabe histórica.

20. El nombre se conserva en la toponimia urbana granadina como Fajalauza. 
«De los negros ${ }^{21}$ ). Es evidente que Tamarid no se había propuesto definir sistemáticamente y con precisión el léxico recopilado, sino tan solo de ofrecer una orientación a sus lectores sobre el significado, omitiendo, desde luego, cualquier dato que pudiera resultar consabido para los hablantes contemporáneos.

La información semántica que ofrece Guadix no presenta similitudes con la contenida en el Compendio más allá de las que pueden ser fruto del azar. En primer lugar, los enunciados definicionales son totalmente independientes, y no solo por la práctica de Guadix de definir con precisión, a modo de un diccionario de lengua, la totalidad de las voces, sino también por la formulación empleada, según nos revela de inmediato cualquier ejemplo elegido al azar: así, mientras Tamarid explica raça como "cepa o rayz", Guadix opta por "casta o linage"; añazea se caracteriza vagamente por el primero como "cosa de plazer", mientras que el segundo define "contentamiento o regozijo que suelen tomar vnos amigos con otros juntándose en alguna güerta o heredad a comer y holgarse"; Tamarid explica alcartaz brevemente como "papelón revuelto", mientras que Guadix ofrece una definición sumamente detallada: "papelón plegado en buelta redonda y figura piramidal en que los especieros, confiteros y voticarios ponen y dan las cossas que venden y se lleuarían mal en la mano, si no fuesen puestas o embueltas en alguna cosa". A esto se suma que a veces los dos autores difieren no solo en cuanto a la forma de sus definiciones, sino incluso en cuanto a su contenido: mientras que Tamarid señala para la voz almadraba los dos valores "pesquera" y "tejar", Guadix solo conoce el primero; para Tamarid, ceutí es "moneda morisca que corría en Çeuta", y para Guadix "una suerte o natío de limones"; para Tamarid, támara es "dátil", mientras que para Guadix es "rama de árbol, assí como se quitó o se cortó del árbol”. Aún más significativa es la no aparición en Guadix de algunas de las definiciones más dudosas de Tamarid, como las que corresponden no a la voz castellana lematizada, sino al presunto étimo árabe (arriate "calçada, camino, o passo"22; cid, o cidi «Es amo o señor»; iazarina "Es cosa de Argel $»^{23}$; bahari «Es halcón que passa la mar»; mameluco «Es hijo del señor»; ceifa «Es la ciega [sic] o cortadura», frente a "espada o alfange" en Guadix). En otros casos, la información semántica de Tamarid no recogida en la Recopilación corresponde a valores semánticos que los lexicógrafos modernos no han podido documentar de forma clara (por ejemplo, alcorán «Es recopilación»; alcoba «Es capilla»; almuédano «Es munidor $[s i c] »)$.

Aparte de la información semántica poco más ofrece Tamarid en la microestructura: cabe mencionar algunos someros comentarios explícitos

21. El término, escasísimamente documentado (para la explicación etimológica véase Corriente 2005: 107), queda aclarado gracias al comentario de Guadix: «Es un mote o baldón que en España dan a los negros o etiopianos diziéndoles que perdonen abuxahamel."

22. El DCECH comenta: «No es fidedigna la ac. "calzada, camino o paso" que atribuye López Tamarid a arriate, pues solo se basa en una etimología falsa; debiera borrarse del diccionario académico.»

23. Guadix, en cambio, define correctamente el significado castellano de lo que él lematiza bajo la var. jazerina: «una suerte de malla o de cotas de malla». 
sobre la etimología, que, no obstante, no pasan de ser una simple adscripción estratigráfica del étimo: trece voces (almizque, almodrote, capón, corral, cozina, dique, gaio, horno, iuglar, marauedi, peregil, pulga, saya) incluidas en el listado se describen como híbridas latino-árabes mediante el comentario «Paresce latino y arábigo", y una se interpreta como vocablo híbrido "teutónico y arábigo" (dique). Tan solo en uno de los casos así marcados, el de perexil, reconoce Guadix igualmente una etimología híbrida, aunque árabe-castellana en lugar de árabe-latina, por lo que podemos descartar cualquier influencia también en cuanto a este elemento.

\section{II.2.2 La microestructura de las entradas dedicadas a nombres de lugar}

La microestructura de la Exposición es generalmente algo más extensa que la de la parte dedicada a apelativos, y, sobre todo, contiene datos más peculiares en el sentido de que reflejan un conocimiento mucho más específico y subjetivo. Básicamente podemos distinguir tres tipos de elementos: las localizaciones geográficas, los significados de los étimos, y las atribuciones de las interpretaciones a otros autores. El primer elemento está presente en 15 de los 76 casos, lo cual contrasta con la constancia absoluta con que se ofrece este tipo de información en la Recopilación. La comparación de los enunciados que contienen las localizaciones geográficas revela que Guadix redactó los suyos con total independencia: así, no recoge información de Tamarid que podía tener interés para los lectores no familiarizados con la microtoponimia granadina como la que ofrece este para Bibarreha-«Es la puerta de la calle de sant Hieronymo» (Guadix se limita a afirmar: «es el nombre de una puerta de la insigne ciudad de Granada»)- o para Bibalhaçarin - «son los Arquillos del Alcaçaua» (Guadix: «nombre de una puerta de la insigne ciudad de Granada»)-; el Campo de Çafayona es localizado por Tamarid «cerca de Granada quatro leguas»; por Guadix, en cambio, «entre Sancta Fee y Las Ventas de Caçín». La lista de hidrónimos de Tamarid figura bajo el título "Ríos de España», sin que se dé información específica acerca de los nombres concretos; Guadix, en cambio, localiza cada nombre con cierta precisión geográfica: así, del río Guadarriçaz afirma que "corre por Sierra Morena, pasando por cerca del pueblo Vilches», y del Guadalete que «naze en las sierras de Çahara y vazia en la mar, en el Puerto de Santa María». Las ocasionales similitudes, por lo demás, pueden ser casuales, ya que ambos autores estaban perfectamente familiarizados con los lugares y los nombres granadinos.

En cuanto al elemento central (y sin duda también más significativo a nuestros efectos) de la microestructura, el etimológico, hemos de destacar, en primer lugar, que Tamarid, a diferencia de Guadix, se limita a reflejar el significado en castellano del étimo en lugar de establecer este de forma explícita. Con frecuencia no ofrece una única interpretación, sino dos, tres, y hasta cuatro diferentes, lo cual explica el hecho de que el texto contenga un total de 87 propuestas etimológicas para un conjunto de 73 nombres. Guadix tan solo recoge 50 de las interpretaciones de la Exposición. Este amplio 
desaprovechamiento de la información de Tamarid ${ }^{24}$ se comprueba de forma plástica en entradas como, por ejemplo, la consagrada a Guadiana, en la que este expone nada menos que cuatro interpretaciones:

El río de Anna, que es su vocablo antiguo, o río de Diana, o río de la fertilidad, o río del llanto.

de las cuales Guadix no recoge sino una, con toda seguridad ampliamente conocida en la época, y añade otra nueva:

Consta de guad, que en arábigo significa río, y de Anas, que es el nombre de una población y rastros de edificios antiguos qu'están en la ribera d'este río, en la parte que llaman Estremadura. De suerte que todo junto, guadanas, significa el río de Anas, .i., el río de aquella población llamada Anas. Parecer a sido de algunos arábigos qu'este nombre consta de guad [...] y de ầana [...]; assí que, todo junto, guadàâna significa río por nosotros o río para nosotros; vale y significa tanto como dezir nuestro río [...].

Dado que resultaría difícil imaginar que Guadix -cuyo afán de recoger una información lo más abundante posible se percibe a lo largo de toda la obrarenunciara a hacer uso de más del $42 \%$ de las interpretaciones de Tamarid, hemos de concluir, una vez más, que, al menos durante la redacción de la Recopilación, no tenía presente materialmente el texto de Tamarid. En este sentido apunta también el que, en alguna ocasión (por ejemplo, s.v. Alixares), Guadix niegue incluso explícitamente la existencia de interpretaciones anteriores, a pesar de que el nombre había sido objeto de interpretación ya en el Compendio ${ }^{25}$ :

Alixares [...] Este nombre a puesto en cuidado a muchos hombres y muy arábigos, $y$ todos an encogido los hombros y callado sus bocas, y no me marauillo de que, aunque muy arábigos, no ayan sabido interpretarlo, porque no es nombre arábigo sino turquesco, .i., de la lengua turquesca.

La misma conclusión se ve reforzada, además, por el hecho de que las explicaciones etimológicas coincidentes en cuanto a su contenido en ningún caso presenten coincidencias claras (y menos aún literales) en cuanto a su forma. Basta con comparar entradas como las dedicadas a nombres como Hiznatorafe o Alhambra para comprobar la falta de dependencia directa entre las dos obras ${ }^{26}$. Si Tamarid ofrece para el primero dos escuetas explicaciones

24. Guadix recoge una de dos interpretaciones en los artículos Bibalbunaytar, Alcaicería, Jabalquinto, Badajoz y Valladolid; una de tres en Gibraltar; una de cuatro en Guadiana; dos de cuatro en Bibalmazán, Darro, Xenil y Madrid. Ignora toda la información etimológica del Compendio en Alixares, Xarragui, Bibarreha, Bisagra, Zocodover, Zacatín, Guadalete, Guadahortuna, El Castillo Locobin, Belmar, Aranjuez, Almodóvar y Calatançón.

25. Donde leemos: "Alixares [...] quiere dezir casas sumptuosas y edificios reales.»

26. Guadix dispone en muchos casos de información acerca de los nombres y de los lugares designados que sobrepasa claramente los escuetos datos que ofrece la Exposición, demostrando así nuevamente su independencia. Así, señala numerosos nombres homónimos o parónimos de otras áreas geográficas, o reseña variantes que coexistían en el habla de la época; un ejemplo ilustrativo es la entrada Guadalhorçe o Guadalherça: «Es en España el nombre de un río que corre por el obispado de Málaga, .i., cerca de la ciudad de Antequera, y es también el nombre 
resumidas en el enunciado "El castillo del tarahe, o del barro", Guadix, que asume como propia la primera, atribuye la segunda a «algunos arábigos», y la expone con palabras muy diferentes:

Hiznatorafe. Es en España pueblo del obispado de Jaén. [...] hiznaltaraf significa el castillo de los taraes o de los tamarices [...]. Parecer a sido de algunos arábigos qu'este nombre es hiznaltorab, que significa el castillo de la tierra o el castillo de tierra, .i. de tapia o tapiería de pura tierra.

Alhambra, a su vez, es interpretado por Tamarid con el siguiente comentario:

Alhambra o quiere dezir alcaçar colorada. O tomó esta denominación de vn rey que vuo en ella, que se llamo Alahmar, que quiere dezir colorado.

Guadix, aunque parte en último término del mismo étimo, formula la interpretación de una manera completamente distinta:

Alhambra. [...] significa la bermeja o la colorada, .i., la rubra; devieron de llamar assí a este real castillo y a el pueblo que digo por el terruño de que son hechos o edificados los edificios dél, que es vna tierra bermeja, no mal terruño para tapias; y no es de maravillar que los árabes llamasen assí a este castillo por el color del terruño de que son los edifficios dél, porque los christianos an hecho lo mesmo...

El hecho de que el 57,4\% de las interpretaciones de Tamarid estén presentes también en Guadix puede explicarse sin necesidad de admitir una explotación directa de la Exposición. En primer lugar, la relativa transparencia de algunos nombres para cualquiera que dispusiera de conocimientos del árabe (pensemos en formas como Bibarrambla, Guadalquivir, Guadalmedina, Gibralfaro o Daraçultán) podía fácilmente dar lugar a que diferentes eruditos coincidieran en sus interpretaciones. En segundo lugar, es más que probable que gran parte de las interpretaciones formaran parte de un fondo colectivo de conocimientos y creencias que gozaban de una cierta tradición. De hecho, son llamativas las reiteradas referencias a eruditos contemporáneos-cuyos nombres, sin embargo, no se señalan explícitamente en el interior del texto- a quienes se atribuye la autoría de tal o cual etimología: así, Tamarid afirma explícitamente que Granada «conforme a la mas commún interpretación quiere dezir cueva de Nata»; acerca de Bibamazán indica que, "côforme a la interpretación de algunos», significa "puerta del hospital de los incurables», aunque él prefiere otra explicación: «Pero mejor, la puerta de la iunta, o congregación»; paralelamente, Bibalbunaitar es "puerta de las eras conforme a la interpretación de algunos», si bien el propio Tamarid considera más adecuada otra distinta: «Mejor la puerta del estandarte, o vanderica pequeña».

Aún mucho más frecuentes son las referencias a opiniones de otros autores en la Recopilación. En no pocos casos la información proporcionada por

de otro río que corre por el arçobispado de Toledo y llega a dar vista y su nombre a unas ventas qu'están cerca dél. Guadalherça [sic] significa el río de la guardia, .i., donde ay guardia o cuerpo de guardia; y corrompido dizen Guadalhorçe, y otros que lo corrompen más dizen Guadalerze, sólo aciertan o hablan con propiedad los que dizen Guadalherza.» 
Guadix nos permite descartar con toda seguridad que se trate de Tamarid: así, cuando reseña tres interpretaciones del nombre Çacatín (çoqaltin "mercado de los higos"; çuaqaltin "mercados de los higos"; alçacatin "los traedores o los portadores") que atribuye explícitamente a otros autores con la habitual fórmula "A avido [grandes] arábigos a quien a parecido que...", sin que ninguna de ellas se corresponda con la expuesta concisamente en la Exposición («[La calle de los] roperos»). Para el nombre Aranjuez establece como étimo alranqchaix "las yeguas del exército o del batallón", advirtiendo, no obstante, que ha habido "grandes arábigos a quien a parescido qu'este nombre [...] es alrandalchaix, que significará el laurel del exército»; Tamarid, en cambio, había interpretado el nombre como "casa de los nogales". Es posible que en algunos casos tales «arábigos» fueran los colegas con los Guadix que colaboró durante su estancia en Roma en la traducción de los evangelios (y que identifica en el colofón a su obra que citamos al comienzo); en otros, sin embargo, el propio Guadix especifica que se trata de arabistas españoles ${ }^{27}$ :

Oýdo e de algunos arábigos d'España dezir qu'el nombre d'este río es guadalhimar, que significa el río del asno, .i., flumen asini, y téngolo por disparate, porque el nombre se le devió de imponer por el color que de ordinario lleua, que es bermejo.

\section{LAS OBRAS DE LóPEZ TAMARID y GUADIX EN SU CONTEXTO}

Resumiendo lo anterior, podemos afirmar que Diego de Guadix, según todos los indicios, no explotó de forma directa la obra de López Tamarid. Ahora bien, el que no tuviera presente el Compendio en el proceso de redacción material de la Recopilación durante su estancia en Roma no significa que no exista una dependencia entre las dos obras. De hecho, sería difícil imaginar que el franciscano desconociese el trabajo precursor de Tamarid, publicado en la primera edición del tan difundido Diccionario de romance en latín de Nebrija que se había realizado en Granada, ciudad en la que vivían ambos en 1585. Tal edición no pudo pasar desapercibida a Diego de Guadix, máxime si contenía una novedad bibliográfica tan de su interés, y siendo su autor López Tamarid, hombre perteneciente al mismo ámbito social y profesional granadino. Admitiendo, pues, que Guadix conocía el Compendio y al menos lo recordaba de haberlo consultado, y teniendo en cuenta la enorme similitud de los objetivos que persiguen ambas obras (la recopilación y explicación etimológica de nombres, tanto comunes como propios, de origen árabe), no cabe duda de que el Compendio constituye la principal fuente de inspiración

27. De nuevo, no se trata de la opinión de Tamarid, pues este había interpretado el hidrónimo Guadalimar como "río colorado". Igualmente la interpretación de Bibalbira como «la puerta del poço, dicha assí por un notable, famoso y muy particular poço que ay cerca d'esta puerta», atribuida nuevamente a "grandes arábigos", ha de proceder de eruditos locales distintos de Tamarid, pues este había relacionado el nombre Eluira con la forma latina Iliberis («que aßí se llamó antiguamente Granada»). 
de la Recopilación. La obra de Tamarid, ciertamente, no pasaba de ser en el fondo una primera aproximación, aun imperfecta e incompleta, y basada en una metodología en estado embrionario, al tema: de hecho, su inclusión como apéndice al diccionario de Nebrija, así como la vaga justificación («...a mí me pareció ponellos aquí al fin deste Vocabulario de Romance para hazerle más vniuersal y prouechoso») que ofrece en el colofón el autor, son hechos que indican que este ni siquiera tuvo muy claro su objetivo inicial, y el desarrollo irregular y fragmentario de la microestructura, así como el vacilante criterio de ordenación de unos materiales recopilados sin sistematicidad, revelan que estamos realmente ante una obra elaborada sin contar con un concepto maduro, y dada a la imprenta sin antes haber sometido el texto a una revisión crítica con el fin de depurarlo y regularizarlo.

En este contexto, el diccionario de Guadix se nos presenta -cual si de un primer esbozo de una obra propia de este se tratase-como desarrollo sistemático y perfeccionamiento del Compendio: por una parte, amplía enormemente el corpus léxico lematizado y lo abre a otras lenguas, y por otra parte sistematiza la microestructura y la completa y enriquece con nuevos elementos ${ }^{28}$. Podríamos sospechar que su afán no solo de perfeccionar una obra anterior, sino también de superarla de forma ostensible y contundente, podría ser fruto de una ambición basada en la rivalidad entre personas que competían en el mismo círculo social y profesional: tal vez Guadix sintiera la necesidad de demostrar su superioridad sobre su colega de la Inquisición granadina, quién sabe si se había sentido menospreciado por la mención expresa de otros eruditos en su lugar en el colofón al Compendio:

Todos estos vocablos fueron communicados con muchos hombres insignes y de mucha erudición en lenguas. Y particularmente fueron vistos y añadidos por los illustres señores y nobles hijos dalgos el capitán Gaspar Maldonado de Salazar, y Lope Fustero, secretario del thesoro de la casa de la moneda de Granada, que como tan curiosos y vistos en varia erudición pudieron dar su parescer.

pues es más que probable que conociese a Tamarid personalmente, y en ese contexto resulta perfectamente imaginable que hubiera debatido los problemas etimológicos con él. Sobre el trasfondo de tal rivalidad se explicaría también su afán, sin duda desmedido ${ }^{29}$, de demostrar sus conocimientos en la materia, al igual que la "ufanía», por emplear el término elegido por Federico Corriente (2005: 94), que rezuman pasajes de la Recopilación como el que reproduzco como muestra (s.v. Granada):

28. Señala explícitamente los étimos, justifica sus interpretaciones con argumentos fonéticos y semántico-referenciales, indica variantes de las formas lematizadas, hace comentarios sobre el uso, enumera derivados y fraseología, introduce información enciclopédica, etc.

29. Pues le lleva a postular numerosas etimologías árabes disparatadas, a menudo para formas que tienen claramente otro origen, según ha demostrado de forma contundente Federico Corriente, para quien el fundamento intelectual de Guadix no era, ni mucho menos, tan sólido como pretendía. 
Este nombre a puesto en cuydado a muchos y muy grandes arábigos, que an desseado saber y declarar lo que significa, y, a mi ver, an dicho muchos y muy grandes yerros.

Naturalmente, tales conjeturas no pueden ser demostradas; lo que, en cambio, está fuera de cualquier duda es que las dos obras que hemos comparado aquí constituyen la manifestación todavía hoy palpable de un vivísimo debate -en el que participó, según hemos visto, un gran número de eruditos- que tuvo lugar en la España de finales del siglo XVI acerca de la etimología árabe de buena parte del léxico de los romances ibéricos y de la toponimia peninsular, un interés que alcanzó sus máximas cotas en Granada. Las causas de este interés son fáciles de comprender: a través de la interpretación etimológica de los elementos léxicos más característicos (por ser elementos específicos y diferenciadores) se confiaba en poder llegar al significado «verdadero» de las palabras, a la raíz de la lengua $y$, a través de ella, de la cultura: el propio Tamarid aspiraba a que su trabajo fuese objeto de estima por parte de «los que verdaderamente tratan de saber las cosas en so origen y rayz, y huyr la ignorancia». En este sentido, ambas obras son manifestación de lo que Umberto Eco (1993: 90) llamó «il furore etimologico» de la época; su peculiaridad consiste en centrarse en el árabe como origen de una parte importante del léxico.

La necesidad de conocer y comprender tales raíces era especialmente imperiosa en una tierra como Granada, donde la cristianización aún no había cumplido cien ańos, y donde todavía compartían el mismo espacio dos culturas que, a pesar de haberse influenciado recíprocamente en profundidad, se percibían como antagónicas: una tierra deseosa de definir su propia identidad -entonces todavía en pleno proceso de formación-y de valorarla adecuadamente y con dignidad. En este contexto cobra un significado especial el hecho de que los eruditos eligieran como camino en la búsqueda de tal identidad el estudio etimológico -científico, en definitiva, según el entendimiento de la época- del léxico árabe incorporado al castellano de la región (especialmente de los topónimos, indesligablemente vinculados a la tierra), por cuanto revela la aceptación y valoración positiva del legado árabe (el cultural, no el religioso, no nos engañemos). Con ello estos eruditos se movían en contra de una poderosa corriente que perseguía la marginación y aun anulación de todo lo relacionado con la cultura árabe, y que se sobrepondría y materializaría con la expulsión, muy pocos años después, de los moriscos de España. 


\section{Bibliografía}

Bajo Pérez Elena / Maíllo Salgado Felipe, «Estudio introductorio», en Guadix 2005: 19-131.

Corominas Joan / Pascual José Antonio, Diccionario crítico etimológico castellano e hispánico. Madrid, Gredos, 1980-1991 [citado como DCECH].

Corriente Federico, Diccionario de arabismos y voces afines en iberorromance. Madrid, Gredos, 1999.

- «Notas lingüísticas acerca de la Recopilación de algunos nombres arábigos de Diego de Guadix", en Estudios de Dialectología Norteafricana y Andalusí, 2005, n 9, p. 93114.

Eco Umberto, La ricerca della lingua perfetta nella cultura europea. Bari, Laterza, 1993.

Guadix Diego de, Recopilación de algunos nombres arábigos que los árabes pusieron a algunas ciudades y a otras muchas cosas. Edición, introducción, notas e índices de Elena Bajo Pérez y Felipe Maíllo Salgado. Gijón, Trea, 2005.

- Diccionario de arabismos. Recopilación de algunos nombres arábigos. Estudio preliminar y edición de María Águeda Moreno. Jaén, Universidad de Jaén, 2007.

López Tamarid Francisco, Compendio de algvnos vocablos arábigos introdvzidos en la lengua castellana en alguna manera corruptos, de que comúnmente usamos. Apéndice a Antonio de Nebrija, Diccionario de romance en latín. Granada, Antonio de Nebrija, 1585.

Moreno Moreno Águeda, «Los arabismos del español (siglos XVI y XVII)», en A. Roldán / R. Escavy / E. Hernández / J. M. Hernández / M. I. López (eds.), Caminos actuales de la historiografía lingüistica. Murcia, Servicio de Publicaciones de la Universidad de Murcia, 2006, p. 1175-1185.

- «Estudio preliminar», en Guadix 2007: XXI-XCIII. 